\title{
Significant Advantages of Composite Materials Attached to Improving Technology and Disadvantages of Fiber Related Mining in Harming Humans' Health
}

\author{
Yimeng Zhu
}

\author{
Sendelta international academy Shenzhen \\ leoymz@126.com
}

\begin{abstract}
Frequent use of the category FRP material, Fiber-reinforced polymer, in new innovation of aviation-related topics left a general positive influence on ordinary people in acknowledging composite materials. The purpose of this essay is to provide a critical and comprehensive understanding of the influence of composite material by briefly reviewing some positive effects of composite materials making progress on recent scientific researches, and negative effects on damaging human appearances, invoking hidden chronic diseases when sediments inside composite material are being disassembled and dispersed. Using a collection of data incorporated with scientific reports as well as comments, and a comparable experiment conducted in a Chinese coal mine in July 2021, this study reveals harmful effects composite sediments could have on human beings. The kept ignorance of composites' damages on the human body like congesting respiration system could cause a slow increase in the number of patients getting pneumoconiosis and other typical respiratory illness. This study answers the question of how do we perceive, examine composite material as a whole. More questions like how do we exactly avoid being affected by those harmful particles contained in composites and how to improve our efficiency on extracting raw materials from geographic sites in the premise of securing labors' health is being placed with high expectations to be solved.
\end{abstract}

Keywords: Composite material, Human health, Fiber-reinforced material

\section{INTRODUCTION}

Composite materials, which are known to be one of the crucial factors that contribute to the development of advanced technology in martial devices and mechanical engineering region, have only been focused by people in recent years. In the past 80 years, composite materials went through a significant change from only serving military uses to a widespread of application which you could see it everywhere in the daily life like architectural structures, modern high-tech equipment, transportation protection. Sole Material usually obtain single skill in one specific aspects. Through compositing two or even more resources, the mixture of material could gain multiple properties to provide the product with high quality in varies of aspects (anti-corrosion, high-resisting, antiaging, fireproofing etc). Nonetheless, it contained problems such as dealing physical health damages, causing varies of cancer and provoking respiratory disease in specific. Indeed, composite materials are considered strong on resisting strength, but one slightly changes in its infrastructure will cause an entirely corruption, disassembly of the whole body. Scholars are attempting to work on methods to enforce composite material itself. The future road of composite material is full of hope, but at the same time, it is also full of blocking and failure in all aspects. The brief process of how one concrete kind of composite is made to reinforce specialty, how composite materials according to their function improve their infrastructure and its advantages alongside the damages it could exert on the human-body will be reviewed in this paper.

\section{THE ANALYSIS OF PRODUCING PROCESS OF COMPOSITE MATERIAL}

\subsection{The background of composite material}

Original of composite material is thought to be first appeared in an ancient country where citizens and merchants would use a straw to enforce their cabin by mixing up with clay and other soil pure materials. Since 
then, structures of the composite materials started to lead people into discovery of methods that can process raw materials for architecture purposes. Unfortunately, due to the suppressing of feudalism, processing techniques did not change much until the second industrial revolution, where people attempted to replace primitive materials in military equipment with fiber involved products. After the ending of WW2, fiber materials were highly noticed by governments in national defenses. In the 1990s, however, materialists started to focus more on allowing fiber reinforced material to gain an advantage by being economic efficient in producing and long-lasting in durability in order to satisfy public demand.

\subsection{The processing of composite material}

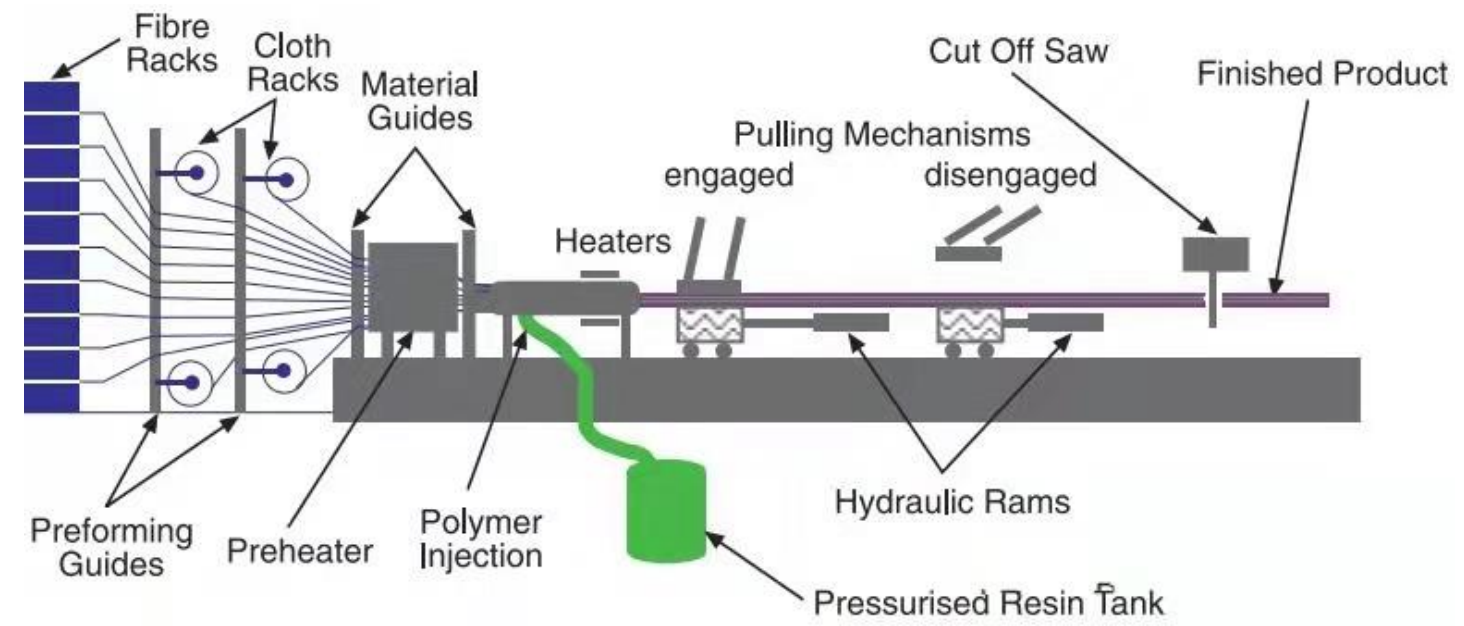

Figure 1 Pultrusion process

\section{THE ADVANTAGES OF PLUTRUSION}

Comparing with the Traditional layering techniques, Pultrusion has outstood its advantages in improving processing and general ability of its products. The first point will be its high use ratio in raw materials. Pultrusion technology can be directly produced using the primary wire and prepreg tape, and the production process does not produce other waste materials except for a small part of the two ends of the profile that needs to be removed. Another point will be pultrusion's supplement to the other two methods of constructing structure. As for winding and braiding, due to the limitation of working principle, they can only produce single-cavity simple section profiles, and it is relatively difficult to arrange longitudinal reinforced fiber. But pultrusion molding technology cannot produce variable section profiles effectively. Pultrusion also obtains high production efficiency as it has two functions of "forming" and "solidifying", which can realize continuous production. Pultrusion speeds of up to $10 \mathrm{~m} / \mathrm{min}$ are unmatched by other form-cure fiber composite production techniques. Through high automatic devices operating in format, it precisely controlled every technological parameter, such
Processing composite material, is to combine raw materials to form a certain structure. Constant crosssectioned FRP structure profile is a result of Pultrusion method. It is a process that will impregnate through the shape of a certain section of the continuous fiber through the mold, in the mold cavity solidification molding or the mold cavity gel, out of the mold heating curing. This method used to produce FRP consists of three main stages under the pulling of the traction of external forces: impregnation, Pultrusion and heat curing. (represented in figure 1) The speed of "pulling" resin impregnated reinforcing fibers and fiber fabrics through a heated curing die will be affected by its profile-complexity, while it is normally at speeds of $120 \mathrm{in} . / \mathrm{min}$. In recent years, Bidirectional, multidirectional woven and stitched fabrics improved mechanical properties in the process[1]. 
results were satisfying since Carbon Fiber displayed surprising characteristics in obtaining various demand the explorer expected to be seen on their product. As Bhatt and Goe point out few facts: 1. Each light and strong material would have a good Strength/weight ratio as steel alloy materials such as Aluminum, titanium, magnesium, carbon and glass fiber; 2. Rigidity or stiffness of a material is measured by its Young Modulus and measures how much a material deflects under stress. Carbon fiber reinforced plastic is over 4 times stiffer than glass reinforced plastic, almost 20 times more than pine, 2.5 times greater than aluminum[2] .Besides strength, Carbon materials are not likely to corrode due to their physics property. Carbon has a powerful structure that keeps it in physical format, which indirectly leads to its high chemical stability. Erosion resistance highlights the importance of using Carbon fiber as coating when aircrafts experience long-term conservation or strike of high PH liquid[3].

\section{POSITIVE APPLICATION OF COMPOSITE MATERIAL}

\subsection{Basic movements of fixed wing aircraft}

Ability to adopt, follow and relieve the force exerted on the body of aircraft is also crucial while the aircraft is climbing and dropping. Elasticity provided by the tensile strength gives the plane chances to adjust the total amount of force experienced on its body. This characteristic is usually displayed in a form of shaking and quivering. Under the proper establishment of framework, Carbon Fiber coating allows it to shrink or expand within a limited range.

\subsection{Carbon fiber on aerial utility}

Selection of the fiber coating polymers also has more options such as the fiberglass and carbon tissues. Each of them has their own advantage and disadvantages as they are processed, build up in different quantities, density and cost. The varies of Carbon material used in the aircraft field indicates that more plans are offered to companies and individuals. Starting from their perspective, diverse option would not only satisfy everyone's demand, but also putting pressure on other peer composite material and consequently, encouraging the further significant science break through.

Focusing on one specific example, a common wing structure of present aircrafts is usually covered with Carbon fiber covers the surface. Although whenever it is being hit by huge strength, it is hard to discover and to fix the Corruption of the structure through outline, it still owns the huge advantage of being light compared to quondam coating.

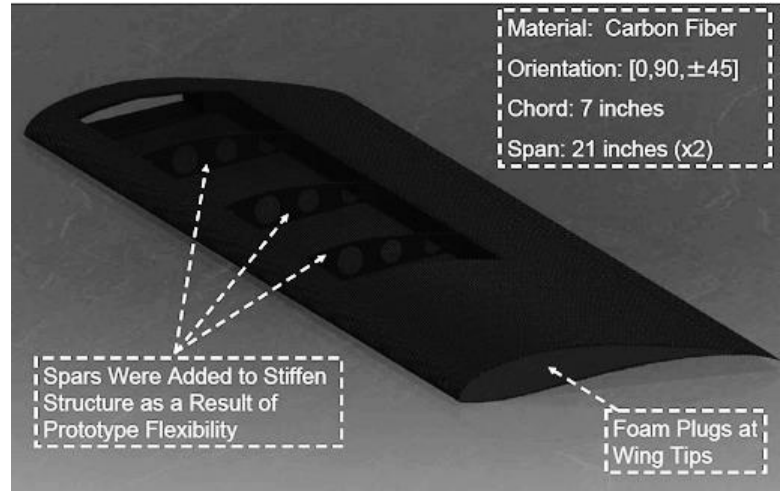

Figure 2 Carbon covered Wing structure

\subsection{Spread of carbon fiber related to human daily}

Carbon fiber being a usual material used in engineering field, had also came across people's life to allow general public to come closer to their own hobbies as they find more practical technique about the material. Because of the universality and reliability of composite materials, more people are willing to understand and promote composite materials to replace traditional materials. Louis joyner published an article sharing his experiences and small tips to help others from plunging into fruitless trying.

Handling light fiberglass cloth isn't easy, but carefully unfolding the cloth onto a smooth surface such as a large piece of illustration board or brown wrapping paper will help reduce snags.

Carbon-fiber cloth comes in a variety of weights; the most usable for FF are approximately 2.25 to 2.9 ounces per square yard. Carbon cloth is considerably stronger and a good deal more expensive then fiberglass cloth.[4]

On 27th October 2017, a list of carcinogens published by the International Agency for Research on Cancer (IARC) of the World Health Organization (WHO) is preliminary collation and reference that asbestos belongs to class A carcinogens, Including its six main kinds: Astrostone, iron stone, amphibole, chrysotile asbestos, crocidolite, tremolite. Most of those materials have been banned by relative country bureaus. Problems of asbestos usually took place both in mining and using. Asbestos is considered as a crucial material utilized in filtration facilities and isolation products. Its physical structure is similar to the composition of fine tubular that is held in standard form[5]. It can be separated into thin asbestos fiber that has a diameter less than one microns. Thus, Bacterial filter material depends largely on this material. As for the blue asbestos, it obtains special chemical toxicants and the ability to purify the air polluted by radioactive particles. However, asbestos are discovered to be easily absorbs and accumulated in human respiratory tract. Long term accumulation will cause 
damage to lungs or even lead to cancer. Workers in the mining factory suffer from huge damages from mining asbestos. By being exposed to high concentrations of fiberglass dust can experience symptoms of upper respiratory irritation and asthma attacks. Fiberglass thickness of $0.5 \mu \mathrm{m}$ and $0.7 \mu \mathrm{m}$ can be generally detected in the lavage fluid of patients' lungs. In addition, there were also changes of bronchiolitis, reticular hyperplasia, and fibrous foci in the lung biopsy.

Asbestos fiber is a general term for certain silicate minerals that can split into elastic filaments with length generally within $3 \sim 50 \mathrm{~mm}$. Using naked eye to observe is difficult to detect. Asbestos fiber can cause asbestosis, pleural mesothelioma and other diseases. Asbestosis is an occupational pneumoconiosis caused by long-term inhalation of asbestos dust with lung interstitial fibrosis as the main lesion. Asbestosis is caused by the deposition of asbestos fibers in the respiratory bronchioles and alveolar walls. In addition, asbestos has toxic effects on macrophages, alveolar epithelial cells and mesothelial cells in lung tissue, leading to fibrosis of lung and pleura[6]. Pathological examination of the patient's eyeball revealed hyperplasia of corneal epithelial cells and increased mucin content in conjunctival fluid, indicating mechanical stimulation[7].

Factories producing chemical fiber had always been concerning about the resources quantity in the whole process which in fact, occupied a large percentage in the total cost. In 2006, multiple experts in Intellectual Ventures Management came along with Bill gates conducted a convention, and discussed the problem of new atomic power generation. Later, under the support of Bill Gates and United States Department of Energy, TerraPower cooperated with Ramaco carbon to search for the possibility of extracting carbon fiber from raw coal. In the near future, coal material will become one of the resources of producing carbon fiber. Coal mining industries will flourish and the limited site, increasing efficiency of coal mining will be highlighted again under the assumption made above. Despite environment restrictions, the mining process separates into unearthing and collecting two parts. This part will focus on the high extent of damages in the stage of collection delt to factory workers.

In coal collection, the process of coal gangue screening is inevitable. Due to the basic same physical properties of gangue and coal, excess impurities and the cost of distinguishing equipment, the screening of coal gangue still needs the cooperation of labor and machine. However, due to the rough collection process and impurities mixed in coal and gangue, the production environment is horrible, which greatly damages the health of workers. To extrude the high concentration of inhalable particles in the working site, Shenzhen Shi Wei company conducted an informal experiment at the XunDong mine in $\mathrm{Xi}$ 'an. The subjects and contents of the experiment were to make a standard adult wear an epidemic mask and breathe normally in different environments, and to make a rough judgment on the severity of the working environment by comparing the appearance color and color of the mask. The experiment was divided into two groups for control experiment. Experiment one required the subjects to stand at the worker's working position and breathe normally. In experiment 2 , the experiment site was changed to xi 'an street, and other variables were fixed. The results showed that a large area of black particles were attached to the upper part of the mask in experiment 1.

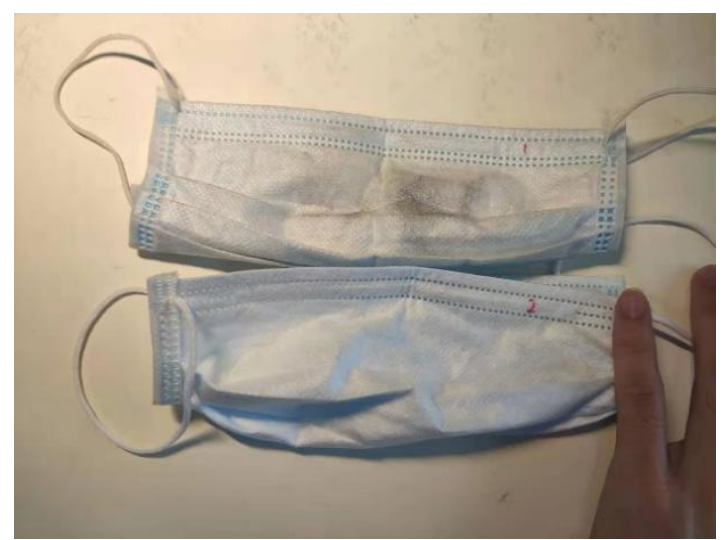

Figure 3 Comparison of contaminated sample 1 and pristine sample 2

Wittenoom in Australia was a huge industrial town that depends its main economy income on the mining, producing of blue asbestos in 1950s. During the time it was Australia's only supplier of blue asbestos. The mine was shut down in 1966 due to unprofitability and growing health concerns from asbestos mining in the area.[8] As a result, more than 2000 out of 20,000 workers and residents of Wittenoom have died from Asbestos Diseases.

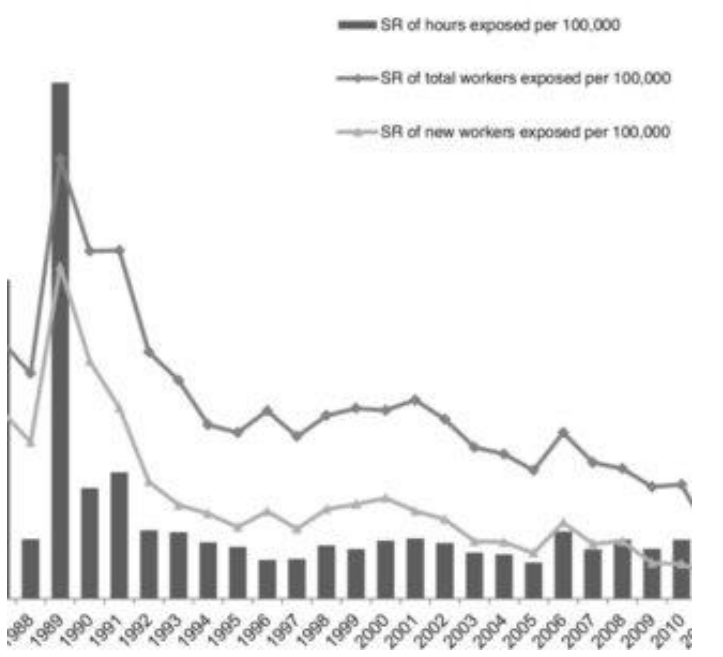

Figure 4 Study results of the time different groups of worker exposed to high concentrated fiber atmosphere in Wittenoom 
In case of contaminating the pollution kept on affecting human-body, Wittenoom was officially removed from the Australia territory where 50,000 hectares $(120,000$ acres $)$ is defined "not capable for human being to live", making it the "largest contaminated site in the southern hemisphere"[9]. Though most of the country has banned the usage of asbestos, many can still be seen in our daily life like the asbestos roof, asbestos cement wall. Once it encounters heavy strike and collapse, air around the region will cause health problem unless the issue is taken proper treated. The lack of control and detection would lead to serious problems when one is adapting a certain resource on machines and engineering facilities.

\section{CONCLUSION}

A short overview around the composite fiber material has been presented to illustrate the function, pros and cons of the fiber material. Increasing amount of material researchers came to find and improve the drawbacks of composite material in order to give a forward development according to their functions individually. Countries like China, United states and Japan have started to work on more efficient new material resources to limit the pollution exerted on both the environment and the human-body. In case of mobbing the superficial coat as well as establishing the infrastructure of the whole product, composite materials acted as a significant influential factor in maintaining the intensity of the building, allowing the continuous floating of air through the fuselage along with the capability of obtaining elastic resistance to face the fierce encounter of airstream and so on. As for I stated above, besides finding new environment-healthy material, specialists are also focusing on finding the solution for former composite influence on human's life. Though, the original conventional metallic material might be replaced in a few decades, its dominant place is still applied in most of the common industry areas nowadays. Development of FRP has been just set off not long ago and there is still a long way for us to discover the most efficient way to apply it on advancing human progress.

\section{REFERENCES}

[1] "Fiber-Reinforced Polymers Composites for Construction-State-of-the-Art biew", C. E. Bakis1 ; L. C. Bank, F.ASCE2 etc

[2] Pooja Bhatt and Alka Goe, Carbon Fibers: Production, Properties and Potential Use

[3] "New construction materials for gliders", Louis Joyner, 2011, $<$ https:/www.modelaviation.com/freeflightmaterial s>
[4] Louis joyner, featured on page 125 in the November 2011 issue. $>$

[5] Carmelita Swiner, MD, 23th November 2020, $<$ https://www.webmd.com/lung/asbestos-exposure $>$

[6] "Biopersistent fiber-induced inflammation and carcinogenesis: Lessons learned from asbestos toward safety of fibrous nanomaterials", Hirotaka Nagai a,b , Shinya Toyokuni a, 17 June 2010

[7] "Characteristics and modifying factors of asbestosinduced oxidative DNA damage", Li Jiang, Hirotaka Nagai etc, September 4, 2008

[8] "Wittenoom residents to be forced out as frustration boils over in effort to close deadly site", Karen Michelmore, 20 ${ }^{\text {th }}$ Mar 2019

[9] "The Wittenoom tragedy", $<$ https://www.asbestosdiseases.org.au/asbestosinfo/the-wittenoom-tragedy/ $>$ May 15th 2017, "Toxicity of synthetic Fibers\& Health", $<$ https://austinpublishinggroup.com/textileengineering/fulltext/arte-v2-id1012.php>

11 August 2017, “Asbestos-Cancer-Causing Substance", february 13, 2019 "Shear Capacity of Fiber-Reinforced Polymer-Strengthened Reinforced Concrete Beams: Fiber Reinforced Polymer Rupture", J. F. Chen1 and J. G. Teng, may 2003 